\title{
Laboratório de inovação na Atenção Primária à Saúde: implementação e desdobramentos
}

\author{
Laboratory for innovation in Primary Health Care: \\ implementation and results
}

Dinaci Vieira Marques Ranzi (https://orcid.org/0000-0002-5404-8195) ${ }^{1}$

Maria Cristina Abrão Nachif (https://orcid.org/0000-0003-3327-4123) ${ }^{1}$

Daniel Ricardo Soranz (https://orcid.org/0000-0002-7224-5854) ${ }^{2}$

Priscila Maria Marcheti (http://orcid.org/0000-0002-1662-4139) ${ }^{3}$

Mara Lisiane de Moraes dos Santos (https://orcid.org/0000-0001-6074-0041) ${ }^{4}$

Alessandro Diogo De Carli (http://orcid.org/0000-0002-4560-4524) ${ }^{5}$
${ }^{1}$ Governo do Estado de Mato Grosso do Sul. Av. Poeta Manoel de Barros, Bloco 8, Parque dos Poderes Governador Pedro Pedrossian. 79031-350 Campo Grande MS Brasil. dinaciranzi0@gmail.com

${ }^{2}$ Centro de Estudos

Estratégicos, Fundação Oswaldo Cruz. Rio de Janeiro RJ Brasil.

${ }^{3}$ Instituto Integrado de

Saúde, Universidade Federal

de Mato Grosso do Sul

(UFMS). Campo Grande

MS Brasil.

${ }^{4}$ Programa de Pós-

Graduação em Saúde da

Família, UFMS. Campo

Grande MS Brasil.

${ }^{5}$ Faculdade de Odontologia,

UFMS. Campo Grande MS

Brasil.

\begin{abstract}
This article presents and problematizes innovation actions aimed at improving the quality of Primary Health Care (PHC), describing an experience that can be adapted to different contexts, considering diverse sociodemographic, economic, cultural and epidemiological realities. We conducted an exploratory study using documentary sources referring to the implementation of the Campo Grande Laboratory for Innovation in Primary Health Care (INOVAAPS). The project proposes the reorientation of the care model adopted in the municipality's public primary care services, redefining work processes and improving the quality of practice. We identified product, process and organizational innovations that have the potential to transform and tailor health care practices to the population's health needs. It is concluded that the proposals implemented by the project focus on the consolidation and expansion of access to primary care, recruitment and training of adequately qualified health professionals, adoption of resolutive technologies, regulatory improvement, and strengthening the mediating role of primary health care.

Key words Innovation, Primary Health Care, Family Health Strategy, Unified Health System, Brazil
\end{abstract}

Resumo O objetivo do estudo foi apresentar e problematizar as ações de inovação para a qualificação da Atenção Primária à Saúde (APS), evidenciando experiência que pode ser adaptada e implementada em diversos contextos, considerando as diferentes realidades sociodemográficas, econômicas, culturais e epidemiológicas. Trata-se de um estudo exploratório realizado a partir de fontes documentais sobre a implementação do Laboratório de Inovação na Atenção Primária à Saúde (INOVAAPS) no município de Campo Grande-MS, que propõe a reorientação do modelo assistencial, com ressignificação dos processos de trabalho na APS, qualificando as práticas. Foram identificadas ações inovadoras organizacionais, processuais e de produto que são potentes para a transformação e readequação de práticas do fazer em saúde. Concluiu-se que as propostas já executadas estão pautadas na expansão, consolidação e ampliação de acesso à APS, à provisão e formação profissionais adequadas, à alocação de tecnologias resolutivas, ao aprimoramento da regulação e à efetivação do papel mediador da APS.

Palavras-chave Inovação, Atenção Primária à Saúde, Estratégia Saúde da Família, Sistema Único de Saúde, Brasil 


\section{Introdução}

Inovar é criar novas realidades, fazer existir algo que não havia ou dar novo sentido a algo que já existia. A inovação é propensa a ser estimulada, promovida e gerida, ganhando força para aplicação de conhecimentos e práticas na área da saú$\mathrm{de}^{1}$, assim como em qualquer outro setor da economia, incluindo serviços governamentais ${ }^{2}$. As análises relacionadas à inovação organizacional repercutem em benefícios diferenciados ${ }^{3}$ e, portanto, trazer a Atenção Primária a Saúde (APS) para este contexto é profícuo no sentido de superar os desafios e implementar mudanças para resolução de lacunas em um setor estratégico e prioritário para o Sistema Único de Saúde (SUS).

Atualmente, o modelo prioritário para implementar a APS no Brasil é a Estratégia Saúde da Família (ESF), que vem recebendo importantes incentivos visando à ampliação da cobertura de atendimento populacional, à reorganização e qualificação dos serviços da atenção primária ${ }^{4}$.

Apesar dos esforços contínuos e evidentes avanços no aumento da cobertura ${ }^{5}$, ainda observam-se importantes lacunas na organização e gestão do SUS, com insuficiente financiamento público ${ }^{6}$, indisponibilidade de equipamentos básicos, variação do perfil dos profissionais, entraves na coordenação da assistência, diferentes modelos de gestão ${ }^{7}$, além de problemas relacionados à infraestrutura, disponibilidade de insumos e qualificação profissional $^{8}$, potencializados pelas diferenças socioeconômicas e desigualdades/iniquidades em saúde.

Outro aspecto que interfere na qualidade da atenção é a qualificação dos profissionais para atuarem na APS $^{9}$. Há evidências de que a formação em saúde da família e comunidade em nível de residência e especialização impactam em melhores indicadores de saúde, com menores índices de internações por causas sensíveis à APS ${ }^{5,10}$, aumento do cadastramento e acompanhamento de pacientes diabéticos e hipertensos ${ }^{5}$, melhoria da atenção e dos indicadores de saúde materno -infantil ${ }^{11}$, e maior orientação aos atributos da APS $^{12}$.

Tais desafios exigem capacidade contínua de inovação na formulação e implantação das políticas públicas e das práticas em saúde no SUS. Para tanto, é necessário investir na formação profissional, na introdução de tecnologias de informação e comunicação ${ }^{13,14}$, na melhoria das condições de trabalho para as equipes e mudanças na dinâmica deste, trazendo os usuários para o centro da atenção e do cuidado ${ }^{4}$.
O processo de inovação deve ser originado da concepção da inovação como uma resposta da organização às pressões do ambiente onde elas atuam ${ }^{3}$. Com essa perspectiva, o projeto do Laboratório de Inovação na Atenção Primária à Saúde (INOVAAPS) foi implementado em Campo Grande-MS com o objetivo de apoiar ações cientificamente embasadas e aplicáveis na prática, principalmente pela inovação de tecnologias assistenciais, de gestão e de comunicação, com o intuito de fortalecer a APS no âmbito do SUS nessa capital.

Frente ao atual contexto político e econômico, marcado pela austeridade fiscal, que fragiliza o princípio da universalidade e corresponde à base das políticas de saúde no Brasil e da premente necessidade de fortalecimento da APS como elemento estruturante organizador do SUS ${ }^{4}$, torna-se imprescindível a realização de estudos que tenham como objeto os processos de inovação na APS, promovendo mudanças na prestação de serviços à população que podem implicar inclusive adequações nas políticas de saúde, tendo como premissa final a melhoria da assistência ofertada ${ }^{3}$.

O objetivo desse estudo foi de apresentar e problematizar as ações do projeto supracitado, colocando em evidência essa experiência como estratégia inovadora para a qualificação da APS, que pode ser adaptada e implementada em diversos contextos, considerando as diferentes realidades sociodemográficas, econômicas, culturais e epidemiológicas.

\section{Método}

Trata-se de um relato de experiência subsidiado por fontes documentais sobre a implementação do Laboratório de Inovação na Atenção Primária à Saúde (INOVAAPS) no município de Campo Grande-MS, no período de março a outubro de 2020. Este estudo apresenta dados originais os quais serão problematizados a fim de permitir aos leitores a associação do que foi planejado e implementado nesse município a outros contextos, a partir da oferta de alternativas de inovação que possam ser disparadoras de outras ações em distintas realidades.

Foram utilizados documentos produzidos ao longo de todo o processo de desenvolvimento do projeto até o mês de outubro de 2020 , que apresentassem e discutissem as ações de inovação do projeto INOVAAPS. Estes compreenderam: a. projeto INOVAAPS; b. relatórios gerenciais; c. informações a partir das apresentações e debates 
do I seminário INOVAAPS Campo Grande-MS (realizado em setembro de 2020); d. informações disponíveis na plataforma do Laboratório INOVAAPS $^{15}$.

Os dados documentais foram organizados e apresentados de modo a explicitar as finalidades e metas do projeto, Unidades de Saúde e equipes da APS participantes. Na sequência, as metas, principais ações e desdobramentos foram apresentadas e relacionadas às recomendações para fortalecimento da APS no Brasil, propostas por Tasca et al. ${ }^{4}$.

\section{Resultados}

O projeto INOVAAPS vem sendo realizado desde o início de 2020 nesta capital, que abriga população de mais de 895.982 pessoas, o que representa cerca de $32 \%$ do total populacional do estado. Com densidade demográfica de 104 hab. $/ \mathrm{km}^{2}$, Campo Grande é o terceiro maior e mais desenvolvido centro urbano da região Centro-Oeste e a 22a maior cidade do Brasil, segundo o IBGE ${ }^{16}$.

O sistema de saúde local é relativamente bem estruturado, em alinhamento com a maioria das diretrizes e políticas do Ministério da Saúde. Representa importante ponto de referência da Rede de Atenção à Saúde (RAS) em nível estadual, dispondo de serviços na APS, Atenção Especializada, Urgência e Emergência, e Atenção Hospitalar. Entretanto, ainda apresenta fragilidades referentes ao cumprimento de atributos da APS, ordenação da formação profissional e cobertura pela ESF.

A partir desta constatação, a Secretaria $\mathrm{Mu}$ nicipal de Saúde de Campo Grande (SESAU) procurou o Ministério da Saúde, por meio da Fundação Oswaldo Cruz (Fiocruz), solicitando apoio para planejar e implementar as mudanças necessárias, tendo em vista as fragilidades supracitadas. Em julho de 2019 celebrou-se um Termo de Cooperação Técnica entre as três instituições, com o objetivo de apoiar o SUS desse município no desenvolvimento de ações estratégicas nas áreas da APS e Vigilância em Saúde. Estas seguem os pressupostos do apoio institucional, articulando experiências e saberes para a identificação, análise e intervenção sobre cenários considerados essenciais para elevar a resolutividade do sistema de saúde local e facilitar a tomada de decisão pelos gestores, tendo como contexto a APS.

O projeto propõe a reorientação do modelo assistencial, com ressignificação dos processos de trabalho na APS, qualificando as práticas, bem como o processo de aprendizagem no cotidiano do trabalho, abrangendo a formação em PósGraduação em saúde - nível de Residência. Desta forma, almeja-se a produção de conhecimentos e tecnologias que contribuam para suprir a lacuna de profissionais com perfil voltado para a APS.

O modelo explicativo-finalístico da proposição das ações estruturantes a serem desenvolvidas pelo projeto está apresentado na Figura 1.

Para tanto, foram elencadas metas prioritárias, elaboradas a partir das demandas locais, apresentadas no Quadro 1.

Os cenários elencados para a implementação das ações do INOVAAPS correspondem a nove Unidades Básicas de Saúde (UBS), as quais foram definidas estrategicamente pela SESAU. Essa determinação ocorreu em função das fragilidades territoriais, da abrangência de cobertura de equipes de APS e da grande densidade populacional dos territórios em que se inserem, contemplando a diversidade dos sete distritos sanitários do $\mathrm{mu}$ nicípio.

As nove UBS participantes do projeto, o número de equipes em 2019 e o número de novas equipes em 2020 em cada uma delas estão apresentadas na Tabela 1. Tais ações impactaram em aumento da cobertura da ESF de $52 \%$ para $75 \%$ após o início do INOVAAPS.

Os resultados em relação às metas, ações e desdobramentos a partir da implementação do Laboratório de Inovação na Atenção Primária a Saúde (INOVAAPS) até o mês de outubro de 2020, e suas interlocuções com as recomendações para fortalecimento da APS no Brasil propostas por Tasca et al. ${ }^{4}$ estão apresentados no Quadro 2.

Ao longo desse processo foram identificadas dificuldades e barreiras para implementação das ações de inovação propostas pelo INOVAAPS. Tais dificuldades são de diversas naturezas, entre as quais consideramos relevante destacar algumas barreiras relacionais, organizacionais e estruturais, todas comuns em processos de mudanças. Observamos a resistência de alguns gestores e profissionais da APS para implementação das mudanças em questão; disputas em relação a hegemonia de projetos e propostas; dificuldades para a articulação da APS com os demais serviços da RAS; um número expressivo de planilhas a serem alimentadas; realização de um número excessivo de reuniões; a inconsistência dos cadastros dos usuários; dificuldades para a implementação da Carteira de Serviços, com incorporação de procedimentos que não eram realizados na APS do município; dificuldades na integração ensino-serviço com a inclusão dos residentes nas 


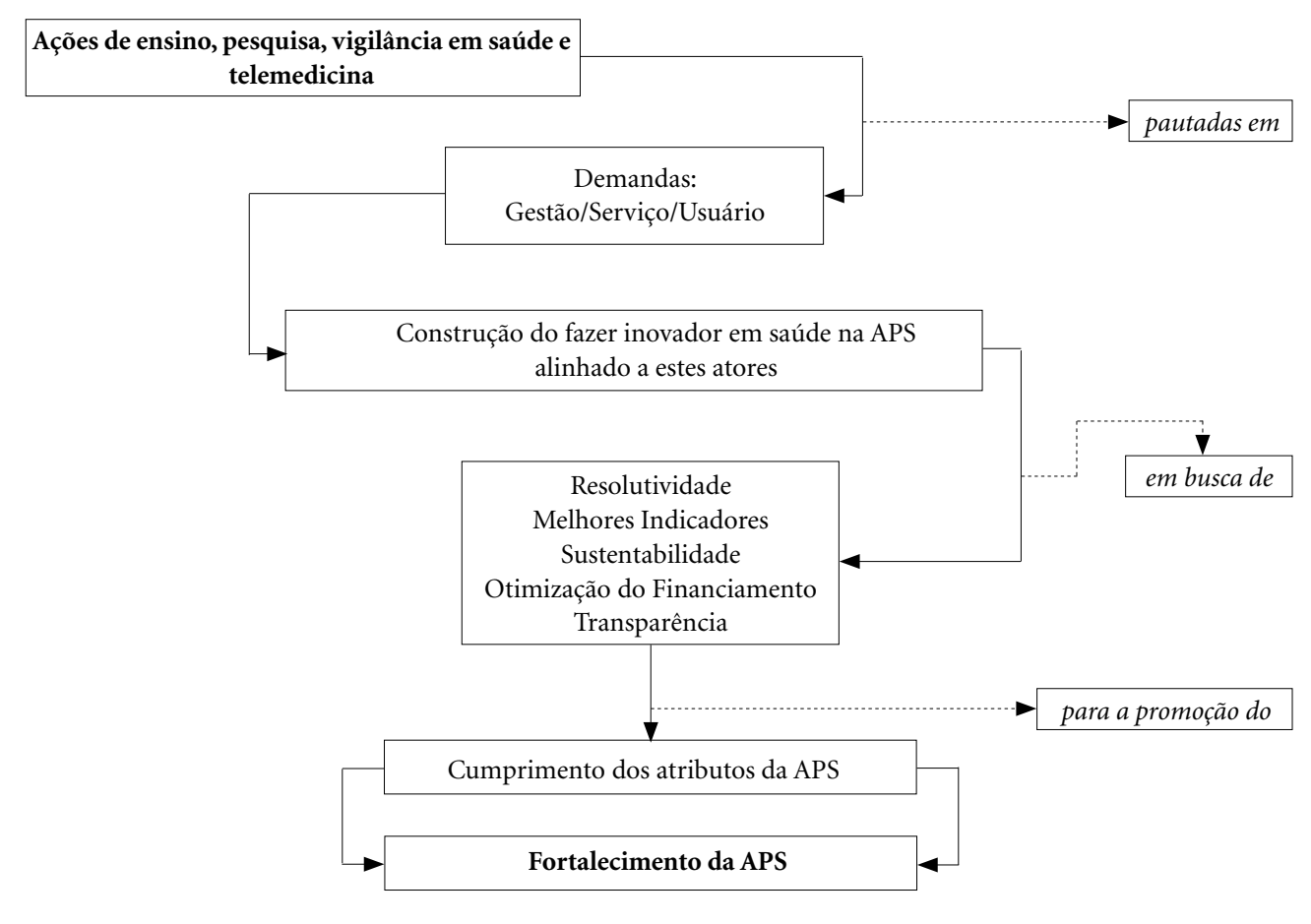

Figura 1. Ações, percurso e finalidade do projeto INOVAAPS.

Fonte: Elaborado pelos autores.

Quadro 1. Metas do Laboratório de Inovação na Atenção Primária a Saúde (INOVAAPS).

- Apoiar a gestão local nos processos de tomada de decisão
- Reestruturar o Programa de Residência em Medicina da Família e Comunidade
- Estruturar o Programa de Residência Multiprofissional em Saúde da Família
- Qualificar as Equipes de Atenção Primária em Saúde
- Implantar o Observatório da Atenção Primária a Saúde de Campo Grande para apoiar as Equipes de Saúde
da Família na gestão do território
- Apoiar as ações estratégicas de Vigilância em Saúde
- Implantar o serviço de telemedicina e apoio diagnóstico
- Realizar pesquisas de avaliação da APS utilizando o PCA Tool
- Realizar pesquisas de opinião com os trabalhadores do SUS

Fonte: Elaborado pelos autores.

equipes de APS; pouca resolutividade do cuidado aos casos mais complexos; dificuldade para o trabalho em equipe; inadequação da estrutura física e ambiência das Unidades de Saúde.

Para o enfrentamento de tais obstáculos, a equipe aposta nas negociações, nos momentos de formação e qualificação (cujas ofertas são diversificadas e frequentes), no protagonismo das equipes para o cuidado centrado no usuário e no território; na busca de alternativas viáveis para resolução dos problemas; na reorganização e otimização do SISREG, na atuação dos apoiadores; na oferta de tecnologias e ferramentas de gestão do cuidado; no apoio matricial, nas ações 
Tabela 1. Unidades Básicas de Saúde selecionadas, número de equipes existentes e novas após INOVAAPS, Campo Grande-MS, 2020.

\begin{tabular}{|c|c|c|c|c|}
\hline Unidade & $\begin{array}{c}\text { No eSF existentes } \\
\text { em } 2019\end{array}$ & $\begin{array}{c}\text { No eSB existentes } \\
\text { em } 2019\end{array}$ & Novas eSF${ }^{\star}$ & Novas $\mathrm{eSB}^{\star \star}$ \\
\hline USF Oliveira & 03 & 03 & 01 & 01 \\
\hline USF Batistão & 03 & 03 & 01 & 01 \\
\hline USF Itamaracá & 04 & 04 & 01 & 01 \\
\hline USF Moreninha & 04 & 04 & 02 & - \\
\hline USF Parque do Sol & 04 & 04 & - & - \\
\hline USF Noroeste & 04 & 04 & - & - \\
\hline USF Vida Nova & 03 & 03 & - & - \\
\hline USF Coophavilla & 04 & 06 & 06 & 04 \\
\hline USF Tiradentes & 04 & 08 & 08 & 06 \\
\hline Total & 33 & 39 & 19 & 13 \\
\hline
\end{tabular}

${ }^{\star}$ Equipes Estratégia Saúde da Família; ${ }^{\star \star}$ Equipes Saúde Bucal.

Fonte: elaborado pelos autores.

Quadro 2. Ações do Laboratório de Inovação na Atenção Primária a Saúde (INOVAAPS) de acordo com as metas preestabelecidas, recomendações para o fortalecimento da APS no Brasil, ações realizadas e desdobramentos.

\begin{tabular}{|c|c|c|}
\hline $\begin{array}{c}\text { Recomendações } \\
(\text { Tasca et al., 2020 })\end{array}$ & $\begin{array}{c}\text { Metas } \\
\text { INOVAAPS }\end{array}$ & Ações e desdobramentos \\
\hline \multirow[t]{2}{*}{$\begin{array}{l}\text { Ofertar ações e } \\
\text { serviços de saúde } \\
\text { de acordo com as } \\
\text { necessidades da } \\
\text { população. }\end{array}$} & \multirow[t]{4}{*}{$\begin{array}{l}\text { Apoiar a } \\
\text { Gestão local } \\
\text { nos processos } \\
\text { de tomada de } \\
\text { decisão. }\end{array}$} & $\begin{array}{l}\text { Produção da Carteira de Serviços da APS. } \\
\text { A Carteira de serviço foi implementada em todas as unidades de } \\
\text { APS do município. Essa medida foi importante para nortear as } \\
\text { ações de saúde na APS do município, com forte reconhecimento da } \\
\text { clínica multiprofissional. Ampliou o escopo de práticas efetivamente } \\
\text { realizado na APS, atendendo as diretrizes do Ministério da Saúde com } \\
\text { redução dos encaminhamentos para atenção especializada e serviços } \\
\text { de urgência. Apresenta-se como uma importante ferramenta de gestão } \\
\text { do cuidado. }\end{array}$ \\
\hline & & $\begin{array}{l}\text { Criação da Caderneta do Usuário para o registro e acompanhamento } \\
\text { dos procedimentos e serviços de saúde. } \\
\text { A Caderneta do Usuário funciona como um passaporte para toda a } \\
\text { rede de saúde, com o registro do caminho percorrido pelo usuário na } \\
\text { RAS do município; contém os agendamentos e demais informações } \\
\text { relativas à saúde do usuário, bem como disponibiliza informações } \\
\text { sobre a organização e ofertas da RAS. }\end{array}$ \\
\hline $\begin{array}{l}\text { Aumentar o } \\
\text { financiamento da } \\
\text { APS. }\end{array}$ & & $\begin{array}{l}\text { Revisão de Termos de Referência de Contratos e Convênios com } \\
\text { prestadores de serviços. } \\
\text { Observou-se que, ao longo do tempo, os valores gastos com um } \\
\text { importante prestador de serviços aumentou substancialmente, e o } \\
\text { número de procedimentos realizados diminuiu. Ou seja, pagava-se } \\
\text { mais, por menos serviços. } \\
\text { Foram renegociados os contratos e convênios, estabelecendo metas } \\
\text { quantitativas e qualitativas. Houve aumento na arrecadação em } \\
\text { função do aumento de produção. Com essa ação houve entrada de } \\
\text { R\$ 7.368.817,97 nos cofres do município, refletindo no aumento de } \\
\text { recursos para a APS. } \\
\text { Ampliação da cobertura de ESF de 52\% para 75\%. } \\
\text { Maior adesão às portarias do Ministério da Saúde e, } \\
\text { consequentemente, maior incremento financeiro para o município e } \\
\text { ampliação do acesso à saúde aos usuários. }\end{array}$ \\
\hline Não se aplica & & $\begin{array}{l}\text { Assessoria Jurídica e Contábil. } \\
\text { O apoio técnico ao gestor possibilita que as decisões e deliberações } \\
\text { sejam estabelecidas com orientação contábil e jurídica. }\end{array}$ \\
\hline
\end{tabular}


Quadro 2. Ações do Laboratório de Inovação na Atenção Primária a Saúde (INOVAAPS) de acordo com as metas preestabelecidas, recomendações para o fortalecimento da APS no Brasil, ações realizadas e desdobramentos.

\begin{tabular}{|c|c|c|}
\hline $\begin{array}{c}\text { Recomendações } \\
\text { (Tasca et al., 2020 })\end{array}$ & $\begin{array}{c}\text { Metas } \\
\text { INOVAAPS }\end{array}$ & Ações e desdobramentos \\
\hline $\begin{array}{l}\text { Garantir estrutura } \\
\text { física e tecnológica } \\
\text { adequadas, } \\
\text { com ambiência, } \\
\text { conforto e } \\
\text { fornecimento } \\
\text { adequado de } \\
\text { insumos para o } \\
\text { funcionamento das } \\
\text { unidades básicas de } \\
\text { saúde. }\end{array}$ & & $\begin{array}{l}\text { Suporte às inovações com a disponibilização de equipamentos } \\
\text { e insumos necessários para a implementação das mudanças } \\
\text { propostas. Kits para os residentes e preceptores, seguro estudantil, kit } \\
\text { instrumental para Unidades de Saúde. } \\
\text { Houve melhorias no processo de trabalho a partir da disponibilização } \\
\text { de materiais para as equipes e para os residentes. Os kits incluem } \\
\text { estetoscópios, esfigmomanômetros, equipamentos de proteção } \\
\text { individual, fitas métricas, lanternas, mochilas, squizes, pendrives, } \\
\text { oxímetros de pulso, peak flow, sonar fetal portátil, doppler vascular, } \\
\text { glicosímetros, tiras reagentes de urinálises, balança digital portátil, } \\
\text { termômetro digital, oto-oftalmoscópio, entre outros. Também foram } \\
\text { disponibilizados livros para a biblioteca, em apoio à formação. } \\
\text { Mapeamento das estruturas físicas das Unidades de Saúde } \\
\text { Levantamento de custos para captação de recursos com objetivo } \\
\text { de adequação da estrutura física das Unidades de Saúde, e } \\
\text { desenvolvimento de planilhas orçamentárias para planejamento das } \\
\text { reformas nestas Unidades. } \\
\text { Desenvolvimento de projetos arquitetônicos para melhoria das } \\
\text { estruturas físicas das Unidades de Saúde da Família. } \\
\text { Elaboração do Manual de Ambiência para as Unidades de Saúde da } \\
\text { Família. } \\
\text { Desenvolvido layout para identificação das UBS conforme } \\
\text { padronização preconizada pelo Ministério da Saúde. } \\
\text { Realizadas algumas adequações das Unidades de Saúde de modo } \\
\text { a atender aos parâmetros de estrutura física e garantia de uma } \\
\text { ambiência adequada, entendida como espaço social e profissional. } \\
\text { Por exemplo, um dos problemas comuns das UBS foi a estrutura } \\
\text { física das farmácias. Foram realizados ajustes nesses espaços de modo } \\
\text { a melhorar a armazenagem dos medicamentos e o atendimento ao } \\
\text { público. E assim, contribuir para o fortalecimento da assistência } \\
\text { farmacêutica e, consequentemente, melhorar a atenção aos usuários. } \\
\text { Reorganização do Almoxarifado da SESAU, com organização } \\
\text { do ambiente, ajustes no processo de trabalho e uniformização } \\
\text { de processos. Controle de entrada e saída de insumos, e } \\
\text { acompanhamento dos prazos de vencimentos. } \\
\text { Tais ações repercutem em melhor alocação dos recursos e materiais, e } \\
\text { menores perdas. }\end{array}$ \\
\hline $\begin{array}{l}\text { Desenvolver } \\
\text { sistema de } \\
\text { regulação centrado } \\
\text { na APS. }\end{array}$ & & $\begin{array}{l}\text { Reorganização do processo de trabalho do sistema de regulação da } \\
\text { SESAU - SISREG - com vistas à integração com a APS. } \\
\text { Médicos da APS fazem o agendamento de procedimentos e consultas } \\
\text { para os outros serviços da Rede de Atenção à Saúde, impactando } \\
\text { em maior agilidade nos agendamentos, redução das filas de espera e } \\
\text { maior equidade no acesso. }\end{array}$ \\
\hline
\end{tabular}

continua

que promovam o encontro e favoreçam a interlocução entre eSF, eSB, equipes de Vigilância em Saúde e, de maneira mais incipiente, entre APS e demais pontos da RAS; no fortalecimento dos
Conselhos Gestores; na melhoria da infraestrutura e ambiência. Ainda, todas as ações que descritas no Quadro 2 contribuem para superação dos desafios. 
Quadro 2. Ações do Laboratório de Inovação na Atenção Primária a Saúde (INOVAAPS) de acordo com as metas preestabelecidas, recomendações para o fortalecimento da APS no Brasil, ações realizadas e desdobramentos.

\begin{tabular}{|c|c|c|}
\hline $\begin{array}{c}\text { Recomendações } \\
\text { (Tasca et al., 2020 })\end{array}$ & $\begin{array}{c}\text { Metas } \\
\text { INOVAAPS }\end{array}$ & Ações e desdobramentos \\
\hline $\begin{array}{l}\text { Qualificar } \\
\text { habilidades dos } \\
\text { profissionais da } \\
\text { APS em relação } \\
\text { à tecnologias do } \\
\text { cuidado. } \\
\text { Ampliar a } \\
\text { atuação clínico- } \\
\text { assistencial de } \\
\text { todas as categorias } \\
\text { profissionais das } \\
\text { equipes de APS, } \\
\text { com a utilização } \\
\text { de protocolos } \\
\text { multiprofissionais } \\
\text { baseados na melhor } \\
\text { evidência cientifica } \\
\text { disponível. }\end{array}$ & $\begin{array}{l}\text { Qualificar as } \\
\text { Equipes de } \\
\text { Atenção Primária } \\
\text { à Saúde. }\end{array}$ & $\begin{array}{l}\text { Realização reuniões de equipe com a participação de todos os } \\
\text { profissionais e residentes; ações formativas como sessões clínicas e } \\
\text { seminários, por meio de encontros presenciais e, durante a pandemia, } \\
\text { virtuais. } \\
\text { As equipes elaboraram diagnóstico situacional dos territórios de suas } \\
\text { responsabilidades. } \\
\text { Qualificação do acolhimento; inserção de todos os profissionais das } \\
\text { equipes na vigilância em saúde no território; implantação da consulta } \\
\text { farmacêutica. } \\
\text { Procedimentos que antes eram realizados na atenção especializada } \\
\text { ou serviços de urgência passaram a ser realizados na APS, como por } \\
\text { exemplo a cantoplastia, lavagem otológica, implantação de DIU, manejo } \\
\text { dos usuários com crises hipertensivas. } \\
\text { Maior resolutividade das ações na APS, com redução do turnover. } \\
\text { Reorganização do processo de trabalho das eSB, com implementação da } \\
\text { coordenação do cuidado compartilhado entre eSF e eSB; construção de } \\
\text { fluxograma de acesso, considerando aspectos clínicos e vulnerabilidade } \\
\text { social, e maior atenção aos usuários que necessitam de visitas e } \\
\text { atendimentos domiciliares; acolhimento a todos que buscam tratamento } \\
\text { odontológico, com avaliação e classificação de risco; planejamento } \\
\text { estratégico participativo; implementação de fluxo integrado com a } \\
\text { eSF para o pré-natal odontológico; apoio às eSB na análise e gestão de } \\
\text { informações - e-SUS e SISREG. } \\
\text { Aumento da produtividade das eSB, com aumento do acesso dos } \\
\text { usuários à saúde bucal. } \\
\text { Avanços na coordenação do cuidado e acompanhamento dos itinerários } \\
\text { terapêuticos dos usuários nos diferentes serviços de SB da rede. } \\
\text { Realização de: Seminário INOVA APS; Ciclo de debates (reuniões } \\
\text { temáticas entre os profissionais); formações de toda a RAS municipal } \\
\text { (atualmente on-line devido à pandemia). } \\
\text { Apresentação e debate sobre os } 7 \text { meses de implantação do INOVAAPS. } \\
\text { Ações foram propostas para revisão e ajustes nos processos de trabalho e } \\
\text { qualificação das equipes. }\end{array}$ \\
\hline $\begin{array}{l}\text { Elaborar plano } \\
\text { de formação } \\
\text { profissional para } \\
\text { a APS. }\end{array}$ & $\begin{array}{l}\text { Reestruturar } \\
\text { o Programa } \\
\text { de Residência } \\
\text { em Medicina } \\
\text { da Família e } \\
\text { Comunidade }\end{array}$ & $\begin{array}{l}\text { Reestruturação da Residência em Medicina da Família e Comunidade, } \\
\text { que ofertava um número pequeno de vagas anuais, e havia pouca } \\
\text { procura. Após a reestruturação houve a oferta de um número } \\
\text { expressivo de vagas, com preceptores qualificados e uma remuneração } \\
\text { diferenciada aos residentes. } \\
\text { Aumento da oferta de médicos qualificados para o trabalho na } \\
\text { APS. São } 38 \text { residentes Médicos de Família e Comunidade, o que } \\
\text { repercutiu no aumento expressivo da cobertura e ampliação do } \\
\text { escopo de práticas na APS. Espera-se que ao final da formação os } \\
\text { residentes tenham à sua disposição uma série de ferramentas para } \\
\text { resolverem } 90 \% \text { dos problemas da população que atendem, tanto } \\
\text { quadros agudos, quanto crônicos, bem como esteja qualificado para a } \\
\text { realização de procedimentos pertinentes à APS. }\end{array}$ \\
\hline
\end{tabular}

continua

\section{Discussão}

Os dados desse estudo demonstram a importância dos movimentos de reorientação de gestão, de práticas e de investimento técnico, científico e financeiro em saúde, mesmo quando se trata de políticas já consolidadas. Isto é relevante especialmente em cenários de diversidade social, 
Quadro 2. Ações do Laboratório de Inovação na Atenção Primária a Saúde (INOVAAPS) de acordo com as metas preestabelecidas, recomendações para o fortalecimento da APS no Brasil, ações realizadas e desdobramentos.

\begin{tabular}{|c|c|c|}
\hline $\begin{array}{c}\text { Recomendações } \\
\text { (Tasca et al., 20204) }\end{array}$ & $\begin{array}{c}\text { Metas } \\
\text { INOVAAPS }\end{array}$ & Ações e desdobramentos \\
\hline $\begin{array}{l}\text { Elaborar plano } \\
\text { de formação } \\
\text { profissional com a } \\
\text { APS. }\end{array}$ & \begin{tabular}{l|} 
Estruturar o \\
Programa de \\
Residência \\
Multiprofissional \\
em Saúde da \\
Família
\end{tabular} & $\begin{array}{l}\text { Implementação da Residência Multiprofissional em Saúde da Família, } \\
\text { com a oferta de um número expressivo de vagas. São } 77 \text { residentes de } \\
7 \text { categorias profissionais inseridos na APS do município. } \\
\text { Os programas de residência multiprofissional e médica dispararam } \\
\text { processos antes ausentes ou incipientes nas UBS, como: } \\
\text { territorialização, acolhimento por equipe, implantação do e-SUS AB, } \\
\text { fortalecimento das consultas de enfermagem, pólo de atendimento } \\
\text { e teleconsulta, reorganização do acesso à Saúde Bucal, consultas } \\
\text { farmacêuticas, retomada das reuniões de equipe, qualificação dos } \\
\text { registros clínicos no prontuário eletrônico, caderneta do usuário, } \\
\text { reabilitação pós-COVID-19. }\end{array}$ \\
\hline Não se aplica & $\begin{array}{l}\text { Apoiar as ações } \\
\text { estratégicas de } \\
\text { Vigilância em } \\
\text { Saúde }\end{array}$ & $\begin{array}{l}\text { Contribuição na elaboração do plano de ação para a Integração da } \\
\text { APS com a Vigilância em Saúde. } \\
\text { Realização de oficinas de integração entre APS e vigilância e } \\
\text { promoção da saúde. } \\
\text { Apoio às regionais de saúde nas ações de Vigilância em Saúde } \\
\text { integradas a Atenção Primária por meio de profissionais } \\
\text { especializados nas ações de vigilância em saúde. } \\
\text { Plataforma para a análise especial dos dados do SINAN em tempo real } \\
\text { com devolutiva a cada eSF ou Unidade Básica de Saúde. }\end{array}$ \\
\hline $\begin{array}{l}\text { Promover } \\
\text { estratégias de defesa } \\
\text { e fortalecimento } \\
\text { da APS, incluindo } \\
\text { produção de } \\
\text { conhecimento } \\
\text { científico e } \\
\text { divulgação de } \\
\text { experiências } \\
\text { inovadoras e } \\
\text { exitosas. }\end{array}$ & \multirow[t]{2}{*}{$\begin{array}{l}\text { Implantar o } \\
\text { Observatório da } \\
\text { Atenção Primária } \\
\text { a Saúde de } \\
\text { Campo Grande } \\
\text { para apoiar } \\
\text { as Equipes de } \\
\text { Saúde da Família } \\
\text { na gestão do } \\
\text { território. }\end{array}$} & $\begin{array}{l}\text { Implementação do Observatório de Tecnologia de Informação e } \\
\text { Comunicação em Sistemas e Serviços de Saúde da cidade de Campo } \\
\text { Grande (OTICS-Campo Grande). } \\
\text { Foi criada uma plataforma de livre acesso na qual estão } \\
\text { disponibilizadas informações detalhadas sobre o INOVAAPS, as } \\
\text { ações, documentos, relatórios, manuais, protocolos, cronograma de } \\
\text { atividades, entre outros. Na plataforma há ainda o recurso “Onde } \\
\text { ser atendido", por meio do qual o usuário, mediante a informação } \\
\text { do endereço, consegue identificar qual é a sua unidade de referência, } \\
\text { endereço, contato e horário de atendimento. } \\
\text { https://labinovaapsfiocruz.com.br/portal/\#/ } \\
\text { Todos materiais dos seminários estão disponibilizados na plataforma. }\end{array}$ \\
\hline $\begin{array}{l}\text { Incentivar o papel } \\
\text { mediador da APS. }\end{array}$ & & $\begin{array}{l}\text { Seleção e capacitação de } 7 \text { apoiadores (1 por distrito sanitário) para } \\
\text { fomentar ações de integração da APS com a Vigilância em Saúde. } \\
\text { Monitoramento dos indicadores de saúde. } \\
\text { Orientação e apoio qualificado aos distritos sanitários de saúde. }\end{array}$ \\
\hline $\begin{array}{l}\text { Ampliar formas de } \\
\text { acesso à APS. }\end{array}$ & $\begin{array}{l}\text { Implantar } \\
\text { o serviço de } \\
\text { telemedicina } \\
\text { e apoio } \\
\text { diagnóstico }\end{array}$ & $\begin{array}{l}\text { Implantação da Teleconsulta e Teleinfectologia em função da } \\
\text { pandemia da COVID-19. } \\
\text { Por meio da Teleconsulta foi ofertada aos usuários a possibilidade de } \\
\text { realização de consultas por telefone. } \\
\text { A Teleinfectologia foi implementada com o objetivo de fornecer } \\
\text { suporte clínico por infectologistas para os profissionais de saúde de } \\
\text { todo o país durante a pandemia da COVID-19. }\end{array}$ \\
\hline
\end{tabular}

econômica e demográfica, permeados por especificidades técnicas, éticas e políticas, que promovem a efetividade da intervenção ${ }^{3}$. Nesse caso, implementar estratégias inovadoras com vistas à otimização de todo o aparato de APS existente em uma capital torna-se ainda mais desafiador, ao reconhecermos o papel central APS como coordenadora do cuidado ${ }^{12}$. Assim sendo, ações nucleadas nesse ponto da rede de atenção refletem nos demais, podendo desencadear processos e desfechos em todos os pontos da RAS.

Considerando o território em questão, marcado por questões inerentes aos grandes centros urbanos brasileiros como o crescimento acelera- 
Quadro 2. Ações do Laboratório de Inovação na Atenção Primária a Saúde (INOVAAPS) de acordo com as metas preestabelecidas, recomendações para o fortalecimento da APS no Brasil, ações realizadas e desdobramentos.

\begin{tabular}{|c|c|c|}
\hline $\begin{array}{c}\text { Recomendações } \\
\text { (Tasca et al., 2020 }\end{array}$ & $\begin{array}{c}\text { Metas } \\
\text { INOVAAPS }\end{array}$ & Ações e desdobramentos \\
\hline \multirow[t]{2}{*}{$\begin{array}{l}\text { Promover } \\
\text { estratégias de defesa } \\
\text { e fortalecimento } \\
\text { da APS, incluindo } \\
\text { produção de } \\
\text { conhecimento } \\
\text { científico e } \\
\text { divulgação de } \\
\text { experiências } \\
\text { inovadoras e } \\
\text { exitosas. }\end{array}$} & $\begin{array}{l}\text { Realizar de } \\
\text { pesquisa de } \\
\text { avaliação da } \\
\text { atenção primária } \\
\text { à saúde com o } \\
\text { instrumento do } \\
\text { PCATool. }\end{array}$ & $\begin{array}{l}\text { Realizada pesquisa "Presença e extensão dos atributos da Atenção } \\
\text { Primária à Saúde desde a experiência dos usuários adultos nos } \\
\text { serviços públicos de Atenção Primária à Saúde no município de } \\
\text { Campo Grande Mato Grosso do Sul", com objetivo de verificar o grau } \\
\text { de orientação à APS junto às equipes do município. } \\
\text { Pesquisa com a primeira fase concluída. Os resultados mostram } \\
\text { escores essencial e geral abaixo de 6,6, com melhores resultados nas } \\
\text { Clínicas da Família em relação às UBSF. Tais resultados demonstram } \\
\text { que ainda há um caminho a ser percorrido no município em busca de } \\
\text { ofertas de serviços com forte orientação à APS. }\end{array}$ \\
\hline & $\begin{array}{l}\text { Realizar } \\
\text { pesquisas de } \\
\text { opinião com os } \\
\text { trabalhadores do } \\
\text { SUS. }\end{array}$ & $\begin{array}{l}\text { Pesquisa de opinião com os trabalhadores do SUS de Campo Grande. } \\
\text { A pesquisa avaliou a percepção dos profissionais em relação a fatores } \\
\text { que influenciam em seu desempenho e motivação. Produziu dados } \\
\text { capazes de identificar questões a serem trabalhadas em longo prazo } \\
\text { a partir de ações para melhoria contínua, com intuito de mapear as } \\
\text { áreas críticas onde haverá a aplicação de ações para melhoria de clima } \\
\text { comunicação, transparência e processos. }\end{array}$ \\
\hline $\begin{array}{l}\text { Ampliar a } \\
\text { atuação clínico- } \\
\text { assistencial de } \\
\text { todas as categorias } \\
\text { profissionais das } \\
\text { equipes de APS, } \\
\text { com a utilização de } \\
\text { protocolos } \\
\text { multiprofissionais } \\
\text { baseados na melhor } \\
\text { evidência cientifica } \\
\text { disponível. } \\
\text { Qualificar } \\
\text { habilidades dos } \\
\text { profissionais de } \\
\text { APS em relação } \\
\text { à comunicação } \\
\text { e tecnologia do } \\
\text { cuidado. }\end{array}$ & $\begin{array}{l}\text { Apoiar a } \\
\text { gestão local } \\
\text { nos processos } \\
\text { de tomada de } \\
\text { decisão. } \\
\text { Apoiar ações } \\
\text { estratégicas de } \\
\text { vigilância em } \\
\text { saúde; } \\
\text { Qualificar as } \\
\text { equipes de APS. }\end{array}$ & $\begin{array}{l}\text { Criação do Polo de Atendimento aos usuários com suspeita de } \\
\text { COVID-19. } \\
\text { Elaboração de proposta para manter a vinculação pessoa a pessoa } \\
\text { em tempos de pandemia do novo Coronavírus: organizar ou manter } \\
\text { o cadastro dos usuários organizados; definir usuários vulneráveis; } \\
\text { atenção especial à saúde mental; organização do contato remoto com } \\
\text { os usuários. } \\
\text { Local específico para atendimento dos usuários COVID-19. } \\
\text { Potencialização da implementação das recomendações da } \\
\text { Organização Mundial da Saúde para o manejo dos usuários durante a } \\
\text { pandemia da COVID-19. }\end{array}$ \\
\hline
\end{tabular}

SESAU=Secretaria de Saúde de Campo Grande-MS; RAS=Rede de Atenção à Saúde; SISREG=Sistema de Regulação de Vagas APS=Atenção Primária à Saúde; eSF=Equipes Saúde da Família; eSB=Equipes Saúde Bucal.

Fonte: elaborado pelos autores.

do, falta de planejamento, desemprego e desigualdades sociais consideráveis, ressaltamos que o acesso aos serviços de saúde continua sendo ponto crítico. Dentre os segmentos populacionais, a persistência destas desigualdades e problemas sociais como um todo interferem sobremaneira no processo saúde-doença das comunidades, em especial no que se refere à cobertura da $\mathrm{ESF}^{17}$.

Assim, nesse quesito, ponderamos como positivos os avanços até então alcançados pelo projeto, tendo em vista os importantes redireciona- mentos que foram implementados com o intuito de ampliar o acesso da população aos serviços de APS. Isto efetivou-se pela oferta de ações e serviços centrados nas necessidades da população ${ }^{4}$, com a adoção de instrumentos inovadores como a Carteira de Serviços da APS e a Caderneta do Usuário. Cabe aqui a problematização de que, a despeito dos riscos corridos e de possíveis entraves enfrentados na proposição de ações pelo projeto, a cobertura de ESF no município aumentou significativamente. Este ganho merece destaque, 
pois ocorreu apesar das dificuldades impostas pelos territórios das capitais, como a estrutura social e a pré-existência de uma rede assistencial desarticulada e embasada em modelos assistenciais tradicionais ${ }^{18}$.

Em contraponto, é necessário refletir que a ampliação da cobertura não necessariamente implica acesso aos serviços. Tratam-se de atributos distintos e necessitam de avaliações específicas, considerando as iniquidades territoriais, principalmente aquelas que envolvem vulnerabilidades e que podem interferir na teoria da equidade reversa ${ }^{19}$.

Avanços foram obtidos também em relação à operacionalização do SISREG, que estava desarticulado da APS, restrito a uma central de regulação. A proposta de descentralização das marcações de consultas foi acatada e os próprios médicos da APS as realizam. Cientes de que ainda há problemas a serem suplantados nesse sentido, e que análises temporais necessitam ser executadas a fim de obtermos dados avaliativos pertinentes, consideramos este um passo promissor, pois abrange importante barreira de acesso aos serviços nos outros níveis de atenção. A perspectiva é de que ocorra em nosso contexto situação semelhante ao já relatado em estudo realizado no Rio de Janeiro, onde, entre 2011 e 2015, observou-se aumento significativo (de 86\%, de 790.091 para 1.469.771) no número de procedimentos, exames e consultas agendados em decorrência da descentralização da regulação ambulatorial para os médicos de família de cada unidade de saúde ${ }^{20}$.

Um problema vivenciado pelas equipes de APS é a precarização do trabalho no que se refere à infraestrutura, ambiência e falta de insumos. Nesse aspecto, ações foram planejadas e já estão sendo instituídas com a finalidade de promover melhorias na estrutura e financiamento das equipes de saúde envolvidas. Assim, procuramos induzir o fortalecimento da APS como prioridade sanitária, investindo-se em infraestrutura e organização dos serviços ${ }^{21}$. Corroborando com isto, sabe-se que o aporte adequado de recursos na APS implica seu fortalecimento, melhor provisão de serviços preventivos em populações mais saudáveis e políticas de saúde indutoras do cuidado primário ${ }^{12}$.

A questão da formação de profissionais com perfil adequado para atuarem na APS constitui uma demanda constante na realidade brasileira e foi tomada como meta prioritária do projeto. Ao privilegiarmos a formação médica e multiprofissional para a APS em nível de residência, investimos esforços em processos de ensino-aprendiza- gem inovadores, realizados nos territórios e para estes, tendo como norte as demandas das comunidades em que se inserem as unidades básicas de saúde participantes. Desse modo, fortalecemos a qualificação de recursos humanos e um plano de formação profissional direcionados para a APS, os quais poderão servir de suporte para o provimento de recursos humanos, minimizando-se o problema da rotatividade ${ }^{4}$.

Além da fixação dos profissionais, a qualificação desses trabalhadores por meio de residências ou cursos de especialização em saúde da família e comunidade impacta de maneira positiva em diferentes e importantes indicadores de saúde. A presença de profissionais qualificados reduziu a internação por causas sensíveis à $\mathrm{APS}^{5,10}$, melhorou o cuidado a usuários com condições crônicas como Diabetes Mellitus e Hipertensão Arterial ${ }^{5}$, melhorou os indicadores de saúde materno-infantil ${ }^{11}$, e impactou na maior orientação aos atributos da $\mathrm{APS}^{20}$.

Ainda, estimulam-se ações de integração entre a APS e a Vigilância em Saúde de modo que a construção do conhecimento seja elaborada não somente de forma conexa aos dados epidemiológicos do território geográfico adscrito, mas considerando-se este enquanto espaço vivo permeado por equipamentos sociais e modos de vida diversos, que refletem ações e reações frente ao processo saúde-doença ${ }^{21}$. Nesse alinhamento, ao se estabelecerem práticas de processos de trabalhos em aproximação ao território, estando o serviço de APS disposto ao engendramento com a comunidade em que se insere, consideramos que serão potencializadas a resolutividade e a satisfação do usuário/trabalhadores. Como consequência, podem ser favorecidos desfechos que viabilizem e otimizem o cumprimento dos atributos da APS, com vistas à ampliação do acesso e uma maior qualidade do cuidado.

Destacamos também que todo o planejamento das ações foi elaborado tendo como embasamento as premissas do apoio institucional. Este dispositivo tem se mostrado relevante como estratégia de (co)gestão produtora de melhorias na atenção à saúde e de transformações nos processos de trabalhos, promovendo a aproximação entre o serviço, trabalhadores e gestão, tendo como suporte, permanentemente, a análise crítica das práticas de cuidado, com vistas à produção local de alternativas ${ }^{22}$ para o enfrentamento de demandas que implicam reorientações nos modos de fazer em saúde.

Isto se torna relevante ao considerarmos que, em nível mundial, a carga global de doenças está 
mudando, ao passo que as iniquidades se acentuam e as estratégias utilizadas no passado são insuficientes nesse contexto ${ }^{23}$. Nesse direcionamento, com o transcorrer do projeto, buscouse a viabilização de novos serviços em função de características sociodemográficas, culturais, econômicas e epidemiológicas da população adscrita. Considerou-se também os elementos mobilizadores e modificadores da coordenação da produção do serviço (técnicas e equipamentos) e a necessidade de readequações de infraestrutura física e ambiência nos locais de trabalho. Dessa forma, afirmamos que, do ponto de vista conceitual, as ações até o momento implementadas abrangem inovações de produto, de processos e organizacionais ${ }^{3}$.

Assim, considerando as ações implementadas e os desdobramentos alcançados até então, conjecturamos que o Laboratório de Inovação na Atenção Primária a Saúde (INOVAAPS) já sedimentou importantes avanços no que se refere à reorientação do modelo assistencial, formação e fixação de profissionais voltados aos pressupostos da APS, aumento da cobertura de ESF e melhoria da integração da RAS. Embora comparações de resultados entre os territórios acompanhados pelos laboratórios de inovação não sejam cabíveis em função das singularidades inerentes aos diferentes contextos em que a ESF se insere, faz-se necessário o compartilhamento de vivências e conhecimentos construídos em processos inovadores. Nesse sentido, em relação às ações inovadoras aqui apresentadas, estas estão alinhadas à experiência do Projeto Brasília Saudável, que apresentou relevantes progressos para o fortalecimento da APS, tendo como objetivo a organização do SUS para o pleno exercício da saúde no Distrito Federal ${ }^{24}$.

Compreendemos que as inovações no campo da APS abrangem várias possibilidades e muito avanço ainda se faz necessário no cenário em questão. Porém, entendemos que iniciativas inovadoras constituem movimentos de "vir a ser" e não se estabelecem “a priori”. Esta reflexão é cabível quando admitimos que, apesar do crescimento de descobertas biomédicas para prevenir, tratar e diagnosticar doenças ocorrido nos últimos anos, houve, comparativamente, pouca inovação em nossos sistemas de saúde para expandir a oferta destas e ampliar acesso e qualidade do cuidado para obtenção de resultados mais equânimes em saúde ${ }^{23}$. Por outro lado, em contexto nacional, há que se considerar que certos avanços podem, ainda, estar situados em um nível mais basal, em que os cuidados na APS são atravessados por uma “...realidade de miséria e de desmonte das políticas sociais, não apenas da saúde..."17. Esta noção se faz importante, pois é inegável que diferentes contextos políticos, organizacionais e sociais interferem, dando mais ou menos potência à implementação e sustentação das mudanças pretendidas pelas políticas ${ }^{25}$.

Reconhecer esta situação é fundamental especialmente no cenário da APS, que foi concebida sob a premissa do alinhamento com a comunidade, sendo a base para a oferta de serviços de saúde de qualidade, com papel crítico para a obtenção de cobertura universal de saúde ${ }^{23}$. Assim, diante da situação socioeconômica atual, especialmente em países em desenvolvimento como o Brasil, a reorientação dos serviços da APS no sentido de implementar inovações alinhadas à realidade local pode ser o fator diferencial para a que haja diminuição das disparidades associadas à privação socioeconômica ${ }^{12}$.

Nesse processo de implantação do projeto estabeleceram-se relações de forças inerentes a qualquer movimento de reorientação de práticas institucionais. Estas operam em campos de disputa, permeando atos relacionais da micropolítica do cuidado em saúde, relações de poder e relações intersubjetivas ${ }^{25}$. Assim, podem se comportar como entraves ou como projeção de modos diversos de pensar e construir possibilidades de inovação no contexto da APS/ESF, que, no Brasil, cumpre seu papel de política redutora de desigualdades de acesso aos serviços de saúde ${ }^{19}$ e se consolida como melhor modelo assistencial, com potencial capacidade de inovação em tecnologias assistenciais de gestão e comunicação ${ }^{4}$.

Portanto, tendo em perspectiva o percurso que ainda deve ser explorado pelo projeto nos próximos anos, ao objetivarmos inovações no contexto da APS, nos inserimos em um campo de buscas, descobertas, experimentação e adoção de novos conhecimentos, processos, formas organizacionais e produtos, para que o novo seja ofertado aos usuários ${ }^{3}$. Isto é crucial em países como o Brasil em que, historicamente, os sistemas de saúde se pautaram pelo cuidado episódico de situações agudas e que são precariamente equipados para atenderem as necessidades da comunidade ao longo de seu ciclo de vida ${ }^{23}$.

Finalmente, é importante destacar que todo esse processo foi atravessado pela pandemia da Síndrome Respiratória Aguda Grave 2 (SarsCov-2), com necessidade de alterações e adaptações das ações planejadas, assim como a elaboração e operacionalização de um plano de ação para o enfrentamento da Covid-19 no municí- 
pio, com protagonismo das equipes envolvidas no INOVAAPS.

\section{Considerações finais}

A experiência aqui apresentada está em processo constante de construção e avaliação, pois entendemos que a intencionalidade da inovação no campo da APS deve ultrapassar os pressupostos de planejamentos normativos e estanques. Assim, tendo em vista a trajetória até então vivenciada pelos diversos atores envolvidos, consideramos que, em termos gerais, os dados aqui apresentados são promissores e têm a potência para disparar desfechos futuros que possam abrir novas frentes de atuação para o fortalecimento da APS. Portanto, concluímos que, em interlocução às recomendações de Tasca et al. ${ }^{4}$, as propostas já executadas estão pautadas, em termos finalísticos, à expansão, consolidação e ampliação de acesso à APS, à provisão e formação profissionais adequadas, à alocação de tecnologias resolutivas, ao aprimoramento da regulação e à efetivação do papel mediador da APS.

\section{Colaboradores}

DVM Ranzi, MCA Nachif e DR Soranz participaram da concepção do artigo. DVM Ranzi, MCA Nachif, PM Marcheti, MLM Santos e AD De Carli participaram da redação do artigo e todos participaram da revisão final. Todos os autores participaram da análise crítica. 


\section{Referências}

1. Plonski GA. Inovação em transformação. Estud Av 2017; 31(90):7-21.

2. Organisation for Economic Co-operation and Development (OECD). European Statistical (Eurostat). Oslo Manual 2018: Guidelines for Collecting, Reporting and Using Data on Innovation. 4th ed. Luxembourg: OECD Publishing, Eurostat; 2019.

3. Nodari CH, Camargo ME, Olea PM, Dorion ECH, Claus SM. The framework of the practice of innovation in primary healthcare: a case study. Cien Saude Colet 2015; 20(10):3073-3086.

4. Tasca R, Massuda A, Carvalho WM, Buchweitz C, Harzheim E. Recomendações para o fortalecimento da atenção primária à saúde no Brasil. Rev Panam Salud Publica 2020; 44:e4.

5. Santos MLM, Zafalon EJ, Bomfim RA, Kodjaoglanian VL, Moraes SHM, Nascimento DDG, Santos CAST, Souza AS, De Carli AD. Impact of distance education on primary health care indicators in central Brazil: An ecological study with time trend analysis. PLoS One 2019; 14(3):e0214485.

6. Massuda A, Hone T, Leles FAG, Castro MC, Atun R. The Brazilian health system at crossroads: progress, crisis and resilience. BMJ Glob Health 2018; 3(4):e000829.

7. Facchini LA, Piccini RX, Tomasi E, Thumé E, Teixeira VA, Silveira DS, Maia MFS, Siqueira FV, Rodrigues MA, Paniz VV, Osório A. Evaluation of the effectiveness of Primary Health Care in South and Northeast Brazil: methodological contributions. Cad Saude Pública 2008; 24(Supl. 1):S159-S172.

8. Zavatini MA, Obreli-Neto PR, Cuman RK. Estratégia saúde da família no tratamento de doenças crônicodegenerativas: avanços e desafios. Rev Gaucha Enferm 2010; 31(4):647-654.

9. Stein AT, Ferri CP. Inovação e avanços em atenção primária no Brasil: novos desafios. Rev Bras Med Fam Comunidade 2017; 12(39):1-4

10. Afonso MPD, Shimizu HE, Merchan-Hamann E, Ramalho WM, Afonso T. Association between hospitalisation for ambulatory care-sensitive conditions and primary health care physician specialisation: a cross-sectional ecological study in Curitiba (Brazil). BMJ Open 2017; 7(12):e015322.

11. Nascimento DDG, Moraes SHM, Santos CAST, Souza AS, Bomfim RA, De Carli AD, Kodjaoglanian VL, Santos MLM, Zafalon EJ. Impact of continuing education on maternal and child health indicators. PLoS One 2020; 15(6):e0235258.

12. Starfield B, Shi L, Macinko J. Contribution of Primary Care to Health Systems and Health. The Milbank Quarterly 2005; 83(3):457-502.

13. Pinto LF, Rocha CMF. Inovações na Atenção Primária em Saúde: o uso de ferramentas de tecnologia de comunicação e informação para apoio à gestão local. Cien Saude Colet 2016; 21(5):1433-1448.

14. Pinto LF, Rocha CMF, Mallmann CL. O uso de blogs como ferramenta de apoio à gestão em saúde no nível local. Cien Saude Colet 2018; 23(10):3287-3296.

15. Laboratório de Inovação na Atenção Primária à Saúde (INOVAAPS). [Internet]. 2020 [acessado 2020 out 20]. Disponível em: https://labinovaapsfiocruz.com. br/portal/\#/.
16. Instituto Brasileiro de Geografia e Estatística (IBGE). Cidades e Estados [Internet]. 2020 [acessado 2020 out 20]. Disponível em: https://www.ibge.gov.br/cidades -e-estados/ms/campo-grande.html.

17. Pitombeira DF, Oliveira LC. Pobreza e desigualdades sociais: tensões entre direitos, austeridade e suas implicações na atenção primária. Cien Saude Colet 2020; 25(5):1699-1708.

18. Giovanella L, Mendonça MHM. Atenção Primária à Saúde. In: Giovanella L, organizadora. Políticas e sistemas de saúde no Brasil. Rio de Janeiro: Editora Fiocruz; 2012. p.493-545.

19. Guimarães RM. A teoria da equidade reversa se aplica na atenção primária à sáude? Evidências de 5564 municípios brasileiros. Rev Panam Salud Publica 2018; 42:1-9.

20. Pinto LF, Soranz D, Scardua MT, Silva IM. A regulação municipal ambulatorial de serviços do Sistema Único de Saúde no Rio de Janeiro: avanços, limites e desafios. Cien Saude Colet 2017; 22(4):1257-1267.

21. Brasil. Ministério da Saúde (MS). Secretaria de Atenção à Saúde. Secretaria de Vigilância em Saúde. Guia Política Nacional de Atenção Básica - Módulo 1: Integração Atenção Básica e Vigilância em Saúde. Brasília: MS; 2018.

22. Pereira CM, Feuerwerker LCM. Apoio em saúde: forças em relação. Rev Psicol Política 2018; 18(42);379398.

23. Schwartz D, Duong D, Adam C, Awoonor-Williams JK, Back D, Bang A, Bang R, Beebe M, Bhatt S, Campbell J, Conteh M, Dimitrova D, Dimovska D, Dossou J-P, Evans T, Gadir M, Islam K, Kasyaba R, Kumar P, Levy C, Oanh TM, Monsef N, Oh J, Otoo N, Palazuelos D, Poh A, Sinha S, Smith C, Stewart B, Thomas C, Tritter B, Varnum P, Weilnau T, Ellner A. Primary care 2030: creating an enabling ecosystem for disruptive primary care models to achieve universal health coverage in low and middle-income countries. Annals Global Health 2020; 86(1):9.

24. Tasca R, Ventura ILS, Borges Vanessa, Leles FAG, Gomes RM, Ribas AN, Carvalho WM, Jimenez JMS. Laboratórios de inovação em saúde: por uma Atenção Primária à Saúde forte no Distrito Federal, Brasil. Cien Saude Colet 2019; 24(6):2021-2030.

25. Merhy EE, Feuerwerker LCM, Santos MLM, Bertussi DC, Baduy RS. Rede Básica, campo de forças e micropolítica: implicações para a gestão e cuidado em saúde. Saúde Debate 2019; 43(esp. 6):70-83.

Artigo apresentado em 26/10/2020

Aprovado em 02/02/2021

Versão final apresentada em 04/02/2021

Editores-chefes: Maria Cecília de Souza Minayo, Romeu Gomes, Antônio Augusto Moura da Silva 
